\title{
FPGA-ORIENTED SECURE DATA PATH DESIGN: IMPLEMENTATION OF A PUBLIC KEY COPROCESSOR
}

\author{
Nele Mentens, Kazuo Sakiyama, Lejla Batina, Ingrid Verbauwhede and Bart Preneel \\ ESAT/SCD-COSIC, \\ Katholieke Universiteit Leuven \\ Kasteelpark Arenberg 10, 3001 Heverlee, Belgium \\ email: $\{$ Nele.Mentens,Kazuo.Sakiyama,Lejla.Batina,Ingrid.Verbauwhede,Bart.Preneel $\}$ \\ @ esat.kuleuven.be
}

\begin{abstract}
This paper introduces a secure FPGA implementation of a coprocessor for public key cryptography. It supports Elliptic Curve Cryptography (ECC) as well as the older RSA standard. When choosing adequate key lengths, RSA and ECC are assumed to be secure from an algorithmic point of view. On the other hand, an implementation of these algorithms should also guarantee side-channel security. This feature does not only cause an inevitable performance degradation, but also an area increase. We overcome these drawbacks by fitting the public key architecture and algorithms into a coprocessor that optimally exploites the dedicated features on a Spartan XC3S4000. Although this is a very low-cost FPGA, the performance results of our implementation meet the requirements of a broad range of high-end applications.
\end{abstract}

\section{INTRODUCTION}

Public Key Cryptography (PKC) is the basis for security in many digital information systems. Network applications such as e-mail, e-commerce and e-banking rely on services like key establishment and digital signatures to ensure confidentiality, authentication, data integrity, etc. In other applications like credit cards, PDAs and cell phones, PKC is used to guarantee embedded security. All these diverse applications require suitable implementation platforms. For some systems a general purpose microprocessor suffices the requirements, but when high performance is the main criterium, cryptographic coprocessors in hardware are indispensable. Examples of high performance applications are ATMs, trusted computing platforms and biometric devices.

The most widely used PKC standard is RSA, which was invented by Rivest, Shamir and Aldeman in 1978 [1]. However, its drawbacks became known when a new standard for PKC arrived in 1985: Elliptic Curve Cryptography (ECC),

Nele Mentens, Kazuo Sakiyama and Lejla Batina are partially funded by FWO (G.0450.04), SCARD, ECRYPT and IBBT. proposed by Miller [2] and Koblitz [3], beats RSA in terms of silicon area and speed. The main reason for this is that ECC offers the same level of security as RSA with much shorter key lengths. For example, an RSA public key length of 1024 bits is equivalent to an ECC public key length of 160 bits. For this reason, research mainly focuses on optimizing ECC implementations. However, because RSA has been widely accepted and used, many embedded applications, for which area or power are not a concern, still use RSA. Nevertheless, in the future many applications will gradually evolve towards ECC, which introduces a kind of changeover period. That is why there is a substantial demand for PKC coprocessors supporting both RSA and ECC.

In the mid 1990s, Paul Kocher et al. introduced the concept of side-channel analysis $[4,5]$. From then on, it was common knowledge that countermeasures needed to be taken for preventing an implementation to leak information through power consumption, timing, ... These countermeasures have an impact on the performance and the area of the implementation. To compensate for this area increase, we have made algorithm and architecture choices that make optimal use of the dedicated multipliers and RAM blocks on the FPGA. This results in a secure implementation on an $\mathrm{XC} 3 \mathrm{~S} 4000$, an FPGA from the low-cost Spartan series of Xilinx.

Section 2 gives an overview of the state-of-the-art in PKC coprocessors, while Sect. 3 briefly introduces RSA, ECC and the underlying field operations stressing on the hardware requirements. The design strategy in Sect. 4.1 explains how a secure solution is obtained by exploiting the dedicated features on the FPGA. The resulting implementation is discussed in Sect. 4.2. Finally, Sect. 5 concludes this paper. 


\section{THE STATE-OF-THE-ART IN PKC COPROCESSORS}

This section places our design in a broader context by listing previous work done in the area of PKC coprocessors for RSA and ECC.

The contribution presented in [6] is combining a systolic array architecture with a Montgomery based RSA implementation, achieving the same notion of scalability as introduced in Tenca and Koç [7]. The optimal bound for Montgomery's parameter $R$ is achieved which, with some savings in hardware, completely omits all reduction steps that are presumed to be vulnerable to side-channel attacks.

More recent hardware implementations of RSA include the work by McIvor et al. [8]. They use Carry Save Adders (CSAs) to perform the large word length additions required for Montgomery multiplication.

The work of Crowe et al. [9] proposed a single architecture for RSA and ECC. A hardware optimized version of Montgomery multiplication is used for modular multiplication. The so-called dual processor can operate in parallel for $\mathrm{ECC}$ or in a pipelined manner for RSA.

Because RSA and ECC consist of finite field operation, the performance and area of their implementations depend heavily on the choice of a suitable multiplier. An overview of some implementation options for Montgomery multiplication is given by Koç et al. in [10].

\section{HARDWARE REQUIREMENTS}

In this section, we elaborate on the algorithms we use to implement the operations in RSA and ECC. The reason for these specific choices of algorithms is part of our design strategy and will therefore be explained in Sect. 4.1. For both RSA and ECC, the most crucial operation is modular multiplication. That is why the last part of this section describes Montgomery's Modular Multiplication (MMM) algorithm.

\subsection{RSA}

In RSA, the main operation for encryption as well as decryption, as stated in [1], is modular exponentiation. In our PKC coprocessor this is performed using the $m$-ary or window method, shown in Algorithm 1, which gives a substantial speed-up over binary algorithms [11]. Here $g$ is an element of a ring with cardinality $N$ (i.e. $N$ is the modulus) and $d$ is a positive integer.

This method requires the precomputation of some powers of $g$, which can be done using Algorithm 2.

In Algorithm 1, the exponent $d$ is evaluated in digits of $m$ bits. For every digit $d_{i}, m$ squarings and one multiplication modulo $N$ are performed. To prevent Simple
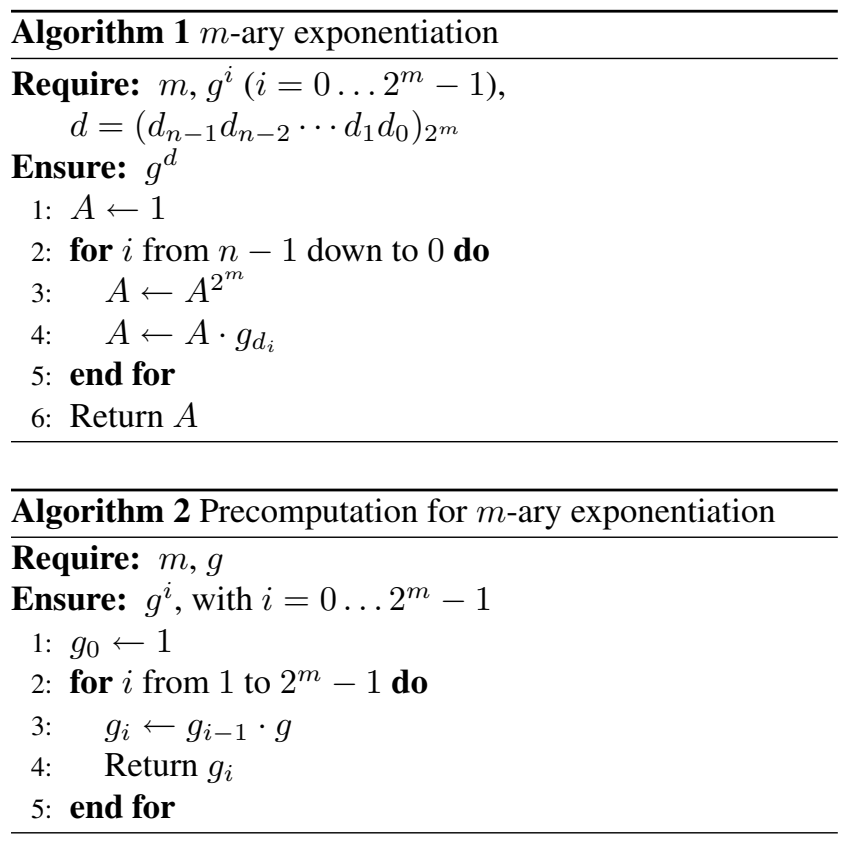

Power Analysis (SPA) attacks [5, 12], the power consumption traces should look similar for every evaluation of $d_{i}$. This is obtained by performing the multiplications and the squarings on the same multiplier instead of using a dedicated squarer. In this way, an attacker cannot distinguish between the operation by looking at the power consumption trace.

To prevent Differential Power Analysis (DPA) attacks [5, 12], which use many power consumption traces together with some statistical analysis, the exponent is "blinded". This countermeasure adds a random multiple of $\varphi(N)$ to the exponent, where $\varphi(N)$ is the Euler totient of the modulus $N$. In [13], more details are given on exponent blinding.

A speed-up for RSA can be achieved using the Chinese Remainder Theorem (CRT) [11]. In this case, modular exponentiation can be performed by two half-size modular exponentiations. Parallelizing these two exponentiations results in a speed-up of a factor approximately 4. Two sequential exponentiations would almost double the speed.

In terms of hardware requirements for RSA, the coprocessor needs a modular multiplier for the exponentiation. Furthermore, it requires RAM blocks for storing the precomputed values $g^{i}$.

\subsection{ECC}

In ECC, the equivalent operation of the modular exponentiation in RSA is point multiplication, which multiplies a point on an elliptic curve with a scalar, resulting in another point on the curve. The scalar serves as the key, while the coordinates of the points contain the data. In our PKC coprocessor, point multiplication is performed using Algorithm 3 [14], 
where $P(x, y)$ is a point on the elliptic curve with coordinates $x$ and $y, k$ is a positive integer and $\mathcal{O}$ is the point at infinity, which serves as the identity element for elliptic curve operations.

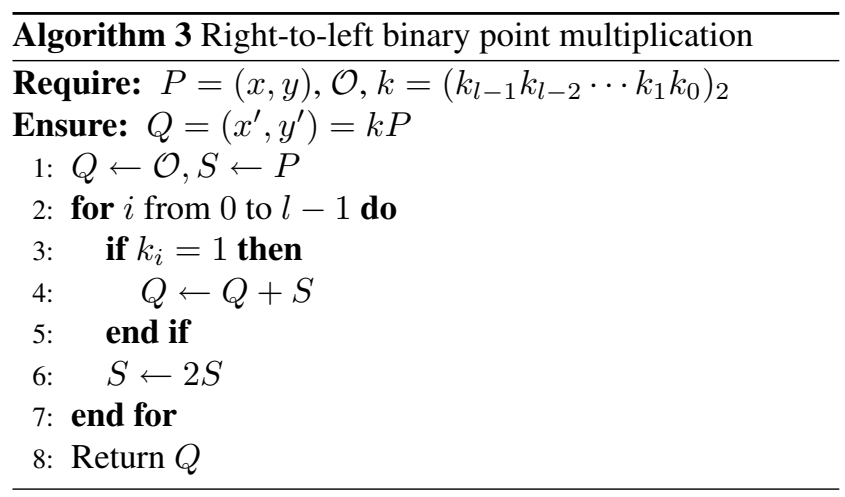

To prevent SPA attacks in ECC, the conditional point addition $Q \leftarrow Q+S$ in Algorithm 3, which is only performed when the evaluated key bit is 1 , has to be avoided. This can be done by inserting a dummy point addition when the key bit is 0 . The result of the dummy point addition will be written to the RAM, but will not be used in the next iteration of the algorithm.

Equivalent to RSA, resistance against DPA attacks can be achieved by key blinding [13]. On top of that, we implemented point randomization as another precaution for preventing DPA attacks [13].

In Algorithm 3 the point operations, i.e. point addition and point doubling, consist of operations in the underlying field. In more detail, a point addition uses 21 field multiplications and 8 field additions, while a point doubling needs 8 field multiplications and 14 field additions [15].

As can be seen in Algorithm 3, the results of point addition and point doubling are written to different intermediate registers $Q$ and $S$. Moreover, there are no data dependencies between both operations, which makes it possible to perform them in parallel in order to double the speed. To guarantee side-channel security, point doubling and addition need to be balanced, i.e. the same finite field operation (modular multiplication or modular addition/subtraction) has to be performed in the same step. This can be achieved by inserting redundant operations in the algorithm for point doubling, resulting in the same sequence of modular multiplications and additions for both operations. This parallel approach leads to the following hardware requirements for the ECC part of our coprocessor: two modular multipliers and two modular adders.

\subsection{Montgomery Multiplication}

The most time and area consuming operation in both RSA and ECC is modular multiplication. To speed this up, we implemented Montgomery multiplication which avoids the trial division that would be needed to perform a straightforward modular multiplication [16]. In the original algorithm a final reduction needs to be performed, but this can be omitted by using a modified version where inputs and outputs are allowed to be bigger than the modulus $N$ [17]. After converting back from Montgomery to normal representation, e.g. at the end of a modular exponentiation, the result is ensured to be smaller than $N$. We adapted this modified algorithm to a version that allows a 16 bit overflow, given in Algorithm 4. The inputs to the algorithm are two finite field elements $a$ and $b$, the modulus $N$, the Montgomery parameter $R=2^{n+16}$ (with $n=$ the number of bits of $N$ ) and $N^{\prime}=-N^{-1} \bmod R$. The result of the Montgomery mul-

Algorithm 4 Montgomery multiplication without final subtraction

Require: $a, b, N, R, N^{\prime}$

Ensure: $a \cdot b \cdot R^{-1} \bmod N$

1: $u \leftarrow a \cdot b \cdot N^{\prime} \bmod R$

2: $t \leftarrow(a \cdot b+u \cdot N) / R$

3: Return $t$

tiplication is the modular multiplication of $a$ and $b$ divided by $R$. To implement Algorithm 4, an integer multiplier and an integer adder are needed.

\section{OUR PKC COPROCESSOR}

In the previous section we elaborated on the hardware requirements for the data path of our coprocessor. The first part of this section focuses on the design strategy. This results in a detailed description of the implementation, which is described in the second part.

\subsection{Design Strategy}

In order to meet the hardware requirements in Sect. 3, the coprocessor needs an integer multiplier and an integer adder.

The multiplier is the most crucial component in terms of area and speed. It is used for performing the integer multiplications in Alg. 4, needed for both RSA and ECC. In addition, RSA using CRT (as explained in Sect. 3) also performs modular multiplications. This means we need to implement a multiplier capable of handling many word lengths: for RSA, a minimum of 1024 bits is required, while ECC needs at least 160 bits. Using CRT for RSA would result in a word length of at least 512 bits.

In order to provide side-channel security, the operations should be balanced as much as possible. Batina et al. elaborate on this in [18]. This means that conditional branches should be avoided at any time and, if possible, parallelism should be introduced to make it harder to distinguish between operations using power consumption traces. 

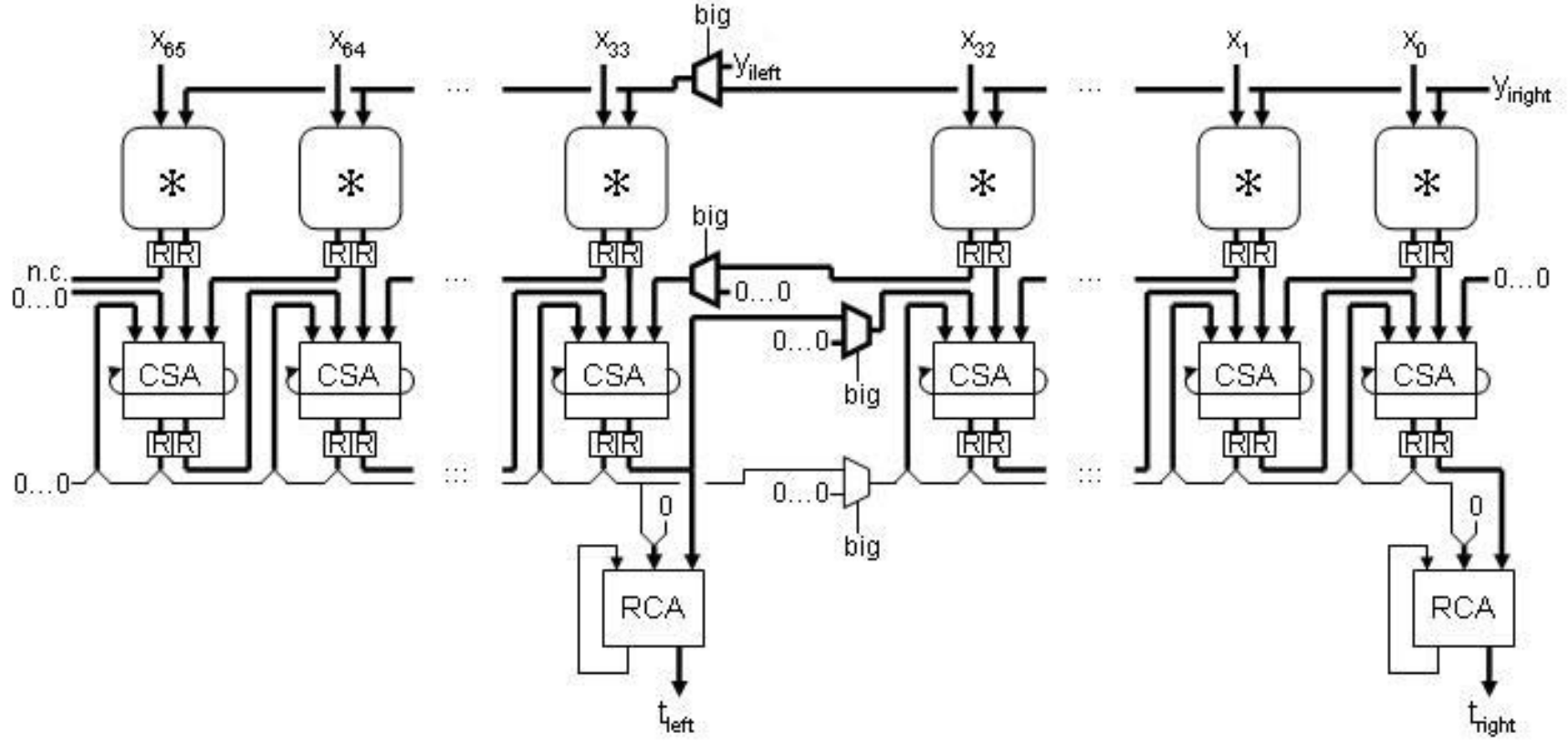

Fig. 1. Multiplier

Because our aim is to implement a public key coprocessor on a low-cost FPGA, like a Spartan, it is not feasible to foresee two parallel 1024 bit multipliers for RSA. That is why we choose for Alg. 1, which uses multiplications in a sequential manner without any conditional branches. For the equivalent operation with CRT, it is possible to use two parallel 512 bit multipliers. Thus, we implement a 1024 bit multiplier that can be configured as two separate 512 bit multipliers. Also for ECC, with a much smaller operand size, parallelism can be exploited. This results in an implementation of Alg. 3, where the problem of the conditional branch is solved by always performing a point addition and a point doubling, independent of the key bit.

In summary, we need a multiplier with three configuration options:

- one 1024 bit multiplication

- two 512 bit multiplications in parallel

- two 256 bit multiplications in parallel

The data path requires a width of 1024 bits, which is quite big. This makes the use of the "free" dedicated features on the FPGA a necessity. The integer multiplications in Alg. 4 can be performed using Fig. 1, where the multiplier blocks at the top are FPGA-dedicated 16x16 unsigned multipliers. The big signal decides on the configuration option. In the parallel option, $t_{\text {left }}$ and $t_{\text {right }}$ are the output signals, while $y_{\text {left }}$ and $y_{\text {right }}$ are the digit-serial inputs. When the multiplier is used for one big multiplication, $t_{\text {right }}$ is the output signal, while $y_{\text {right }}$ is the digit-serial input.

As explained in Sect. 3, at least one overflow bit is needed to implement the Montgomery algorithm without final reduction. Because we use 16x16 multipliers, we extended this idea to 16 overflow bits, resulting in Alg. 4. Because the dedicated multipliers are "free", this does not lead to a substantial area overhead compared to an ASIC-oriented data path with only one overflow bit. Applying this technique to the three configuration modes, leads to a multiplier with an operand width of 1056 bits (determined by the second mode, requiring two $512+16$ multiplications in parallel). This results in a total of $6616 \times 16$ multipliers, which made us decide to use a Spartan XC3S4000 with an availability of 96 dedicated multipliers. Besides dedicated multiplier blocks, our integer multiplier also contains 66 Carry-Save Adders (CSAs) and 2 Ripple Carry Adders (RCAs).

As can be seen from Fig. 1, one of the operands is provided in parallel $(X)$, while the other one is inserted in a sequential manner $(Y)$. This leads to Fig. 2, which depicts 


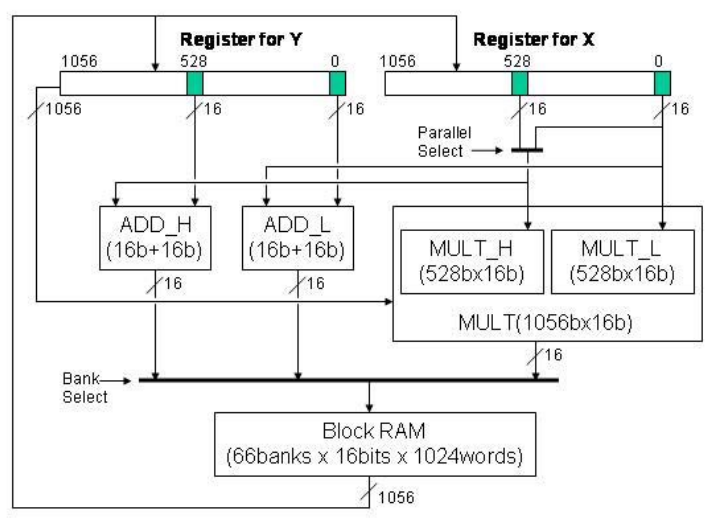

Fig. 2. Data path

the complete data path. To perform point doubling and point addition for ECC in parallel, two ripple-carry adders are implemented. To share the input registers between the adders and the multiplier, the adders use the same data path width of 16 bits. Figure 2 also shows that a RAM bank is used to store intermediate values. This RAM bank consists of 66 dedicated RAM blocks with a width of 16 bits and a depth of 1024. All blocks are controlled by the same address value, but can be enabled separately. This makes it possible to have them storing the 16-bit outputs of the multiplier in a correct way.

To reduce the number of multiplexors, the data path only allows two multiplications or two additions in parallel. An addition in parallel with a multiplication is not possible. The algorithms for point doubling and point addition in ECC, which consist of many modular multiplications and additions, are rewritten for this specific architecture.

In summary, this paragraph shows that the data path is designed with side-channel resistance awareness. This is achieved by balancing the algorithms for RSA and ECC. The next paragraph explains which algorithms are implemented and gives some implementation results.

\subsection{Functional Description}

The coprocessor does not only provide modular exponentiation for RSA and point multiplication for ECC. It also gives the user the possibility to access the lower-level functions, such as modular multiplication, integer multiplication, etc. The hierarchy of operations is shown in Fig. 3, where level 1 corresponds to the lowest level and level 4 to the top level. The lowest level is the only one that interfaces with the data path, while higher levels only communicate with lower levels in the hierarchy. This strategy reduces the number of

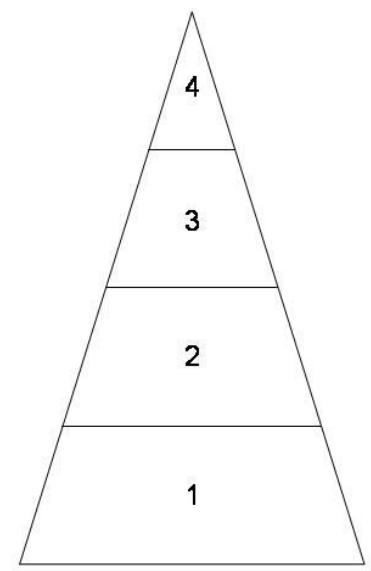

RSA-CRT,

point multiplication

modular exponentiation, modular inversion. point addition, point doubling

modular multiplication, modular addition/subtraction, modular reduction

multiplication, addition/subtraction

Fig. 3. Hierarchy of instructions

multiplexors needed to access the data path. The user can access these operations by an 8-bit command value, $\mathrm{cmd}$, together with a start signal. A valid output signal indicates that the result of the operation is ready. The inputs to the operations have to be written to fixed RAM addresses and the output can be read from a fixed RAM address.

In more detail, the coprocessor provides the following functions:

1. - $256,512,1024,2048$ bit multiplication

- 256, 512, 1024, 2048, 4096 bit addition / subtraction

2. $256,512,1024,2048,4096$ bit modular addition / subtraction

- 256, 512, 1024, 2048, 4096 bit modular multiplication

- $1024 \rightarrow 512,2048 \rightarrow 1024,4096 \rightarrow 2048$ bit modular reduction

3. - 512, 1024, 2048, 4096 bit modular exponentiation (RSA)

- 256 bit point doubling/addition

4. 160,256 bit point multiplication

- 1024, 2048, 4096 bit RSA-CRT

The main contribution of this work is in terms of sidechannel security. RSA and RSA-CRT are implemented without conditional branches (to prevent SPA attacks) and with key blinding (to prevent DPA attacks). ECC is implemented with balanced algorithms (to prevent SPA attacks) and with key blinding and point randomization (to prevent DPA attacks). 
The final result is a secure PKC coprocessor that runs at a frequency of $66 \mathrm{MHz}$ and occupies $57 \%$ of the slices, 66 dedicated multipliers and 66 RAM blocks of a Xilinx Spar$\tan$ XC3S4000 FPGA. Although the focus of our coprocessor is on secure design using dedicated FPGA features, we still give the latency of three main functions:

- 1024 bit RSA: $11.1 \mathrm{~ms}$

- 1024 bit RSA-CRT: $4.0 \mathrm{~ms}$

- 160 bit ECC: $17.4 \mathrm{~ms}$

\section{CONCLUSIONS}

In this paper we present an FPGA implementation of a coprocessor for public key cryptography. In order to obtain side-channel security, the algorithms for RSA and ECC are balanced. Accordingly, the data path has to support these side-channel precautions, which affect the area of the implementation. To compensate for this area increase, we made optimal use of the dedicated multipliers and RAM blocks on a Spartan XC3S4000 FPGA. The resulting performance meets the requirements of a broad range of applications.

\section{REFERENCES}

[1] R. L. Rivest, A. Shamir, and L. M. Adleman, "A method for obtaining digital signatures and public-key cryptosystems," Communications of the ACM, vol. 21, no. 2, pp. 120-126, 1978.

[2] V. Miller, "Uses of elliptic curves in cryptography," in Advances in Cryptology: Proceedings of CRYPTO, ser. Lecture Notes in Computer Science, H. C. Williams, Ed., no. 218. Springer-Verlag, 1985, pp. 417-426.

[3] N. Koblitz, "Elliptic curve cryptosystem," Math. Comp., vol. 48, pp. 203-209, 1987.

[4] P. Kocher, "Timing attacks on implementations of DiffieHellman, RSA, DSS and other systems," in Advances in Cryptology: Proceedings of CRYPTO'96, ser. Lecture Notes in Computer Science, N. Koblitz, Ed., no. 1109. Santa Barbara, CA, USA: Springer-Verlag, August 18-22 1996, pp. 104-113.

[5] P. Kocher, J. Jaffe, and B. Jun, "Differential power analysis," in Advances in Cryptology: Proceedings of CRYPTO, ser. Lecture Notes in Computer Science, M. Wiener, Ed., no. 1666. Santa Barbara, CA, USA: Springer-Verlag, August 15-19 1999, pp. 388-397.

[6] L. Batina, G. Bruin-Muurling, and S. Örs, "Flexible hardware design for RSA and elliptic curve cryptosystems," in In Topics in Cryptology - CT-RSA - The Cryptographers' Track at the RSA Conference, ser. Lecture Notes in Computer Science, T. Okamoto, Ed., vol. 2964. Springer-Verlag, 2004, pp. 250-263.
[7] A. Tenca and Ç.K. Koç, "A scalable architecture for Montgomery multiplication," in Proceedings of 1st International Workshop on Cryptographic Hardware and Embedded Systems (CHES), ser. Lecture Notes in Computer Science, Ç.K. Koç and C. Paar, Eds., no. 1717. Worcester, Massachusetts, USA: Springer-Verlag, August 12-13 1999, pp. 94-108.

[8] C. McIvor, M. McLoone, J. McCanny, A. Daly, and W. Marnane, "Fast Montgomery Modular Multiplication and RSA Cryptographic Processor Architectures," in Proceedings of 37th Annual Asilomar Conference on Signals, Systems and Computers, November 2003, pp. 379-384.

[9] F. Crowe, A. Daly, and W. Marnane, "A Scalable Dual Mode Arithmetic Unit for Public Key Cryptosystems," in Proc. IEEE International Conference Conference on Information Technology - ITCC'05, Las Vegas, 2005, pp. 568-573.

[10] Ç. K. Koç, T. Acar, and B. S. Kaliski, "Analyzing and comparing montgomery multiplication algorithms," IEEE Micro, vol. 16, pp. 26-33, 1996.

[11] A. J. Menezes, P. C. van Oorschot, and S. A. Vanstone, Handbook of Applied Cryptography. CRC Press, 1997.

[12] P. Kocher, J. Jaffe, and B. Jun, "Introduction to differential power analysis and related attacks," http://www. cryptography.com/dpa/technical, 1998.

[13] J.-S. Coron, "Resistance against differential power analysis for elliptic curve cryptosystems," in Proceedings of 1st International Workshop on Cryptographic Hardware and Embedded Systems (CHES), ser. Lecture Notes in Computer Science, Ç. K. Koç and C. Paar, Eds., no. 1717. Worcester, Massachusetts, USA: Springer-Verlag, August 12-13 1999, pp. 292-302.

[14] A. J. Menezes, Elliptic Curve Public Key Cryptosystems. Kluwer Academic Publishers, 1993.

[15] I. Blake, G. Seroussi, and N. P. Smart, Elliptic Curves in Cryptography, ser. London Mathematical Society Lecture Note Series. Cambridge University Press, 1999.

[16] P. Montgomery, "Modular multiplication without trial division," Mathematics of Computation, vol. Vol. 44, pp. 519521, 1985.

[17] C. D. Walter, "Montgomery exponentiation needs no final subtraction," Electronic letters, vol. 35, no. 21, pp. 18311832, October 1999.

[18] L. Batina, N. Mentens, B. Preneel, and I. Verbauwhede, "Balanced point operations for side-channel protection of elliptic curve cryptography," IEE Proceedings - Information Security, vol. 152(1), pp. 57-65, 2005. 